\title{
A Scenario Adaptive Model for Predicting Error Rates in Moving Target Selection on Smartphones
}

\author{
Yawen Zheng \\ Shandong University \\ Jinan, China \\ 201914816@mail.sdu.edu.cn
}

\author{
Jin Huang* \\ Institute of Software, Chinese \\ Academy of Sciences \\ Beijing, China
}

\author{
Juan Liu \\ Shandong University \\ Weihai, China
}

\author{
Chenglei Yang \\ Shandong University \\ Jinan, China
}

\author{
Feng Tian \\ Institute of Software, Chinese \\ Academy of Sciences \\ Beijing, China
}

\begin{abstract}
Modeling error rates in moving target selection is critical in guiding the design and improving user performance in the user interface with dynamic contents. Despite the high accuracy of existing models in predicting error rates for various inputs, they need to fit a large number of samples for a specific interaction scenario. This paper presents a scenario adaptive model that can quickly learn the characterizes of endpoints from small data in a specific scenario, and then accurately predict the error rates in this scenario. We report on two studies evaluating the adaptability of the model in specific users (children and the older user) and pointing postures (walking and one-handed) with a smartphone. Significant improvements in error rates prediction of our model were observed compared to the previous non-adaptive one. We conclude with the findings from our results for insights into future interface design.
\end{abstract}

\section{CCS CONCEPTS}

- Human-centered computing $\rightarrow$ HCI theory, concepts and models.

\section{KEYWORDS}

Moving Target Selection; Adaptive Model; Error Rate; Pointing Uncertainty

ACM Reference Format:

Yawen Zheng, Jin Huang*, Juan Liu, Chenglei Yang, and Feng Tian. 2021. A Scenario Adaptive Model for Predicting Error Rates in Moving Target Selection on Smartphones. In 23rd International Conference on Mobile Human-Computer Interaction (MobileHCI '21), September 27-October 1, 2021, Toulouse \& Virtual, France. ACM, New York, NY, USA, 15 pages. https://doi.org/10.1145/3447526.3472049

\section{INTRODUCTION}

Moving target selection is a common yet challenging task $[13,16]$. Users must keep track of the moving target while also planning the

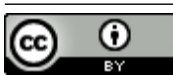

This work is licensed under a Creative Commons Attribution International 4.0 License.

MobileHCI '21, September 27-October 1, 2021, Toulouse \& Virtual, France (c) 2021 Copyright held by the owner/author(s). Publication rights licensed to ACM. ACM ISBN 978-1-4503-8328-8/21/09.

https://doi.org/10.1145/3447526.3472049 timing for selection. Such a behavior necessitates a high level of sensory-motor control ability. Compared with static target selection, acquiring moving targets often result in longer task completion time and higher error rates.

Selecting moving targets in the touchscreen context could be more challenging as issues of user age $[2,10,34]$, the fat finger problem [6], and pointing posture $[9,26]$ further confound fingerpointing. One way to address such challenges is to model and predict the user performance in moving target selection, as it can help us to understand user behavior and optimize our system design.

Previous evidence showed that physiological differences [2], usage habits [26], and situational impairments [5] could lead to significant differences in pointing behaviors in mobile devices. To take into account the multitude of factors that must be considered when modeling the moving target selection in touchscreen contexts, this paper explores a scenario adaptive model to modeling endpoint distribution in moving target selection on smartphones.

One of the latest methods for modeling endpoint distribution in moving target selection is known as the Ternary-Gaussian model [16, 17]. The Ternary-Gaussian model assumes the endpoint distribution is a Gaussian distribution consisted of three Gaussian components, generated from uncertainties of the input device, target size, and target speed:

$$
\begin{gathered}
X \sim N(\mu, \Sigma) \\
\mu=\left(\begin{array}{c}
\mu_{x} \\
\mu_{y}
\end{array}\right)=\left(\begin{array}{c}
a_{x}+b_{x} V+c_{x} W \\
0
\end{array}\right) \\
\Sigma=\left(\begin{array}{cc}
\sigma_{x}^{2} & 0 \\
0 & \sigma_{y}^{2}
\end{array}\right)=\left(\begin{array}{cc}
d_{x}+e_{x} V^{2}+f_{x} W^{2}+g_{x} \frac{V}{W} & 0 \\
0 & d_{y}+e_{y} V^{2}+f_{y} W^{2}
\end{array}\right)
\end{gathered}
$$

Where $W$ and $V$ represent the size and speed of a circular target respectively. $a_{x}, b_{x}, c_{x}, d_{x}, e_{x}, f_{x}, g_{x}, d_{y}, e_{y}$, and $f_{y}$ are empirically determined constants. The Ternary-Gaussian model does not directly predict the error rates; however, with the endpoint distribution predicted by the model, it is easy to obtain the error rates via a multivariate normal cumulative distribution function (CDF) $[16,17]$.

To fit the coefficients of the above the Ternary-Gaussian model, we need to obtain the actual Gaussian parameters (i.e., $\mu$ and $\Sigma$ ) of several target conditions (i.e., target size $W \times$ target speed $V$ ) via user experiments. For example, [17] used a dataset that contains 
16 pairs of actual Gaussian parameters corresponding to $16 \mathrm{~W} \times V$ target conditions to fit the model. For each $W \times V$ condition, pointing endpoints were used to estimate the Gaussian parameters in that condition.

The Ternary-Gaussian model shows high fitting accuracy in modeling endpoint distributions for 1D moving targets [16], 2D moving targets [17], and crossing-based moving targets[15]. It cannot guarantee to be a universal model for all circumstances; that is, the Ternary-Gaussian model fitted to a general dataset may need to be adapted to a specific smartphone-based user circumstance. For example, the Ternary-Gaussian model fitted by data collected from the general population may be inaccurate in predicting the endpoint distribution for an older user on smartphones. A model that can quickly adapt to any specific user circumstance is of great significance to the research and application of pointing uncertainty in moving target selection on smartphones.

We propose an Adaptive Ternary-Gaussian model that combines the specific characteristics from the dataset observed in a specific user circumstance (e.g., specific user groups or specific pointing postures), with the inherent attribute of moving target selection contained in the general dataset. The model is constructed by two steps: First, build a dynamically updated Gaussian parameter dataset, which contains estimated $\mu$ and $\Sigma$ to a specific user circumstance; second, use the dynamically updated dataset to fit a 2D Ternary-Gaussian model. In building the dynamically updated dataset, we combine each newly observed endpoint in the specific user circumstance with Gaussian parameters estimated by a similar model. The similar model represents a model trained by a cluster of endpoints in the general dataset that is similar to the specific user circumstance in form of pointing distribution. With the increase of newly observed endpoints from the specific user circumstance, our adaptive model should approach the actual model of the circumstance. Meanwhile, when only a few endpoints are observed at the beginning, the adaptive model can ensure that it is as similar as possible to the specific user circumstance without losing the inherent attribute of moving target selection from the general dataset.

To evaluate our model, we conducted two user studies. In Study 1 , we conducted an experiment on moving target pointing on smartphones with specific user groups, including 12 older users (average age $=72.7$ ) and 12 children (average age $=4.4$ ). In an offline analysis of predicted touch accuracy, our adaptive model reduced the mean absolute error (MAE) of the predicted error rate by $15.1 \%$ and $14.8 \%$ over the general 2D Ternary-Gaussian model, respectively. In Study 2, we further tested our model under 4 different usage scenarios, including pointing on smartphones when sitting $\times$ two-handed, sitting $\times$ one-handed, walking $\times$ two-handed, and walking $\times$ one-handed. We observed a significantly lower MAE in the predicted selection error rate than the general model with an average reduction of $7.72 \%$.

The contributions of this work are three folds:

- We propose an Adaptive Ternary-Gaussian model to predict error rates under specific user circumstances, which can quickly learn the characteristics of selection endpoints from small datasets.

- Two user studies were conducted, showing that our model not only works well for users with lower motor functions but also has a good performance in adapting to different pointing postures.

- We also contribute a set of findings that may shed light on the future design of user interfaces involving dynamic content: (1) The user's motor ability measured by the throughput (TP) in Fitts' law is highly correlated to the pointing accuracy of moving target selection in a smartphone. The accuracy rate of users with low motor ability is lower than that of the general population.

(2) Compared to the general population, endpoint distributions of children and the older user group show higher absolute uncertainty and motion uncertainty, which indicates that they have lower stability in pointing at moving targets. (3) Compared to the general population, children and the older user group rely more on target size to compensate for the shifting effect ${ }^{1}$ of endpoints.

(4) Comparing the endpoint distributions of children and the older user group, the older user group showed a larger shifting effect while the children have higher absolute uncertainties.

(5) Compared to sitting $\times$ two-handed posture, walking and one-handed posture introduced higher uncertainty in the endpoint distribution.

(6) Most interestingly, comparing the two walking cases, endpoint distributions in one-handed posture have a higher absolute uncertainty while the two-handed posture has a greater shifting effect on endpoints.

\section{RELATED WORK}

We summarize the existing work into three aspects: models for moving target selection, user factors in pointing uncertainty and adaptive interaction technology.

\subsection{Models for Moving Target Selection}

Target selection is a basic task in the user interface. As dynamic contents are becoming ubiquitous in today's user interface, understanding and modeling moving target selection have become one of the hot topics in HCI research [3, 16, 17, 21, 22, 32].

There are two main categories of models for moving target selection including models for movement time and models for pointing uncertainty. In modeling movement time, Jagacinski et al. [19] proposed a model that extended Fitts' Law by taking the factor of target speed into account. The model showed high prediction accuracy in two types of systems including the position control system and the velocity control system. Following Jagacinski et al.'s work, Hoffman [14] presented a model for capture time estimation of moving targets by introducing a steady-state position error. The model was tested with the data of Jagacinski et al.'s work and was found to give an excellent fit. By extending Hoffman's model, Hajri [3] presented a predictor of movement time for moving target selection in 2D space. These movement time models have shown important guidance in user interface design $[3,13]$.

\footnotetext{
${ }^{1}$ The effect of selection endpoints shift in the opposite direction of the target motion [16]
} 
Besides the movement time models, this paper is more interested in another category of modeling works, which focus on predicting the pointing uncertainty in moving target selection. Modeling pointing uncertainty is also helpful to the design of dynamic user interfaces as they can not only reveal the underlying pattern of human behaviors but also predict pointing error rate in target selection [6, 16, 21]. Lee et al. proposed a model in [21] for predicting the error rate of moving target acquisition by integrating the perception process of visual cues into a Temporal Model [22], which mainly interprets the internal cues of humans for timing. Park et al. [32] explored the process of user's click planning and execution process in-depth and proposed an Intermittent Click Planning model to predict the pointing error rates. Instead of transforming the problem of pointing uncertainty into temporal accuracy, Bi et al. [6] derived an endpoint distribution model for static targets based on a dual-Gaussian hypothesis. The dual-Gaussian hypothesis assumes that the endpoint distribution consists of two Gaussian components including a component that corresponds to absolute accuracy and the other one corresponds to target size. Huang et al. [16] extended this work and build a Ternary-Gaussian model to describe the endpoint distribution in moving target selection by introducing another Gaussian component that corresponds to target motion. The Ternary-Gaussian model was later be extended into 2D space [17] and crossing-based task [15] after its proposal.

Despite the effectiveness of the Ternary-Gaussian model in predicting pointing uncertainty and the pointing error rates, there are at least two issues that limit the applications of this model in realworld interactive systems: Firstly, coefficients of the model in one circumstance can not adapt to another.A study in [17] showed very different model coefficients in different devices, which indicated that the coefficients in a specified situation, for example, a specified population or a specified operating posture, can not be adopted to another. Secondly, to estimate the coefficients of the model, a large number of endpoint data must be collected via user experiments. A study in [17] used 16 pairs of actual Gaussian parameters estimated from 2080 endpoints to fit the model for one device. Since the coefficients of the model in one circumstance can not adapt to another, we have to collect data again when the model is applied to a new scenario. Therefore, in this paper, we propose an adaptive model that can quickly learn the characterizes of endpoints from small samples in a specific user circumstance to address the above issues.

\subsection{User Factors on Touchscreen Pointing Accuracy}

Previous studies show different user groups [2], motor abilities [29], situational impairments [31], and postures [26] lead to different interaction behaviors on touchscreen.

Abdouni et al. [2] study aging and gender effects on touch gestures and tactile perception. They found tactile perception of humans weakens with age, while women have better static and active tactile perception than men until 40 years old. Gabyzon et al. [10] evaluate the hand performance abilities necessary for operating a touchscreen of the aging population. The results show vary with ages, the decline in body movement and cognitive function can make pointing targets more difficult. The ability of young people and old people to successfully operate the touchscreen is different, which is related to disease and age. Another recent study, conducted by Sultana et al. [34], also found that older people take a longer time to select targets on a small touchscreen, encounter more difficulties with a higher pointing error rate. Besides the older people, children's touch interactions are also more variable than adults' [42]. Children were found to be less accurate when using touchscreen devices [4], and children's selection endpoints on touchscreen have higher offsets from the target center [35, 36].

When interacting with mobile devices, users with low motor ability, such as those with motor impairments or situational obstacles, will encounter challenges resulting in poor target selection performance [29]. Irwin et al. [18] found that there is a difference in performance between users with motor control impairments and non-disabled users in tap tasks on the touchscreen. The accuracy and duration are both affected by motor control impairments. In addition, Alexander et al. [31] shows that situational impairments such as moving or being encumbered (e.g., while carrying items) have a negative impact on the accuracy of touch gestures, especially difficult to interact with single-finger tap and swipe.

Users prefer to use mobile devices in different postures depending on tasks and environments. Study [26] shows that subjects do not use a uniform posture to interact with smartphone. However, these different body postures also introduce great differences in user performances within the same device. Eardley et al. [9] found that the body posture of lying down leads to more smartphones movement than sitting and standing. Schildbach et al. [33] analyzed the negative effect of walking on target selection and reading when interacting with mobile devices. Lin et al. [23] also demonstrate that in the case of distracting walking, the accuracy of pointing target on touchscreen is significantly reduced. By incorporating the user's posture such as gait into our interaction design, the interaction efficiency could be further improved as shown in [30]. Mayer et al. [25] showed that the effect of vertical acceleration caused by road bumps have a significant effect on the accuracy of touch input. They developed compensatory models to reduce the predicted touch offset.

Among all these factors that affect the user's behaviors in using interactive systems on the touchscreen, we consider validating our model in two of the most common factors associated with age and posture.

\subsection{Adaptive Interaction Technology}

Ability-based design $[40,41]$ is to consider how to make the system perceive and adapt to the user's ability, rather than let the user comply with the established computer system.

Extensive works have been conducted to dynamically adapt our user interfaces to the user's ability, including the user's ability that may be affected by the user factors we mentioned in the last section. Biswas and Langdon [7] designed a model according to the user's hand strength, which improves the user's pointing accuracy on different devices. Montague et al. [27] introduced specific tap-gesture recognizers that use probabilistic to adapt to the variances of touch performances of individuals with motor impairments. Wacharamanotham et al. [37] proposed a swabbing method to improve the accuracy of target selection for older users suffering from hand 
tremors. Martez et al. [28] developed a template matching algorithm Smart Touch, allowing users to use any part of the hand to point on target comfortably and naturally. To address situational impairment, Kane et al. [20] proposed a walking user interface to expand the target maintaining the user's touch accuracy when walking. Another example is the WalkType, developed by Goel et al. [11], which uses the data of accelerometers in mobile phones to improve text input.

Closely related to this paper, adaptive user behavior models are proposed to improve pointing accuracy in mobile devices. Weir et al. [38] use a non-parametric Gaussian process regression to improve touch accuracy. However, this approach needs additional 200 samples to work, which makes it impossible to switch between users and usages. Buschek et al. [8] proposed a flexible framework to adapt and apply user-specific offset models from one device and user to others. However, they didn't further study the variability between users. Mott and Wobbrock [29] created Cluster Touch, a user-specific model that needs only 20 touch samples to quickly calibrate users' touch in their smartphone. Endpoints of the 20 touch samples are used to adjust the touch center of the targets so to improve selection accuracy for different users adaptively. However, the above-mentioned approaches are for static targets, they cannot be directly applied to moving targets.

Inspired by the Cluster Touch [29], we consider building an adaptive model that can quickly adjust its estimated endpoint distribution with a small newly observed dataset.

\section{ADAPTIVE TERNARY-GAUSSIAN MODEL}

To simplify the description of the proposed model, we outline the following important concepts in the model:

- Model Coefficients - the empirically determined constants $a_{x}, b_{x}, c_{x}, d_{x}, e_{x}, f_{x}, g_{x}, d_{y}, e_{y}$, and $f_{y}$ of the 2D TernaryGaussian model described in Equation (1) to (3). It is found that the coefficients in the model can be used to reflect the characteristics of different devices. For example, the higher systematic uncertainty of indirect pointing devices such as computer mouse can be revealed by the coefficient corresponds to absolute uncertainty in the model [17].

- Gaussian Parameters - The $\mu$ and $\Sigma$ that determine a Gaussian distribution in a specified target condition. To fit the 2D Ternary-Gaussian model, a set of actual Gaussian Parameters is required. Generally, no less than 9 pairs of $\mu$ and $\Sigma$ corresponding to $9 W \times V$ conditions are needed.

- Endpoint - The $\mathrm{x}$ and $\mathrm{y}$ coordinates when a user point on a target in a velocity coordinate system. The velocity coordinate system is a coordinate that uses the center of the target as the origin, the direction parallel to the target's moving direction as the $\mathrm{x}$-axis, and the direction perpendicular to the target's moving direction as the y-axis [17]. To fit the 2D Ternary-Gaussian model in practice, we need to first collect endpoints for targets under different $W \times V$ conditions, and then estimate the Gaussian Parameters of each condition with the collected endpoints. At least 100 endpoints are needed to estimate the parameters of a bivariate Gaussian distribution.
- General Dataset - A dataset that contains endpoint data in the general situation. A general dataset usually has adequate endpoints that cover a wider range of target conditions.

- Newly Observed Dataset - A dataset that contains endpoint data in a specific user circumstance. A newly observed dataset usually has very few endpoints scattered in a limited range of target conditions.

An intuitive idea to make the model adapt to the specific user circumstance is to fit the coefficients of the model with the newly observed dataset. However, one of the biggest challenges of doing so is fitting the model with a small number of endpoints. As we mentioned above the original 2D Ternary-Gaussian model requires at least 9 target condition $\times 100$ endpoints for each condition $=900$ endpoints. To address this challenge, we establish a Dynamically Updated Distribution Dataset (DUDDS) to fit the 2D Ternary-Gaussian model adapted to the user. DUDDS contains the estimated Gaussian parameters under multiple target conditions for the target user circumstance.

The idea of constructing the DUDDS is to use a few newly observed endpoints in a specific user circumstance to adjust outputs of a similar model which produces Gaussian parameters similar to the actual model under the specific user circumstance. The similar model is a model tainted by a cluster of endpoints in the general dataset that similar to the specific user circumstance in form of pointing distribution.

There are three steps for constructing the DUDDS as shown in Figure 1: 1) Search for a subset in the general data that is most similar to the newly observed dataset; train an initial 2D TernaryGaussian model with the subset, and called it the similar model (Figure 1 (a)). 2) Use the similar model to produce enough Gaussian parameters (Figure 1 (b)). 3) Adjust the produced Gaussian parameters by linearly combining them with the corresponding newly observed endpoints in a specific user circumstance. By repeating the (2) and (3) steps through enough newly observed endpoints (e.g., 20 endpoints), we are very likely to have a DUDDS that contains enough Gaussian parameters (Figure 1 (c)). Given the DUDDS, we can train a new 2D Ternary-Gaussian model that is supposed to adapt to the specific user circumstance (Figure 1 (d)).

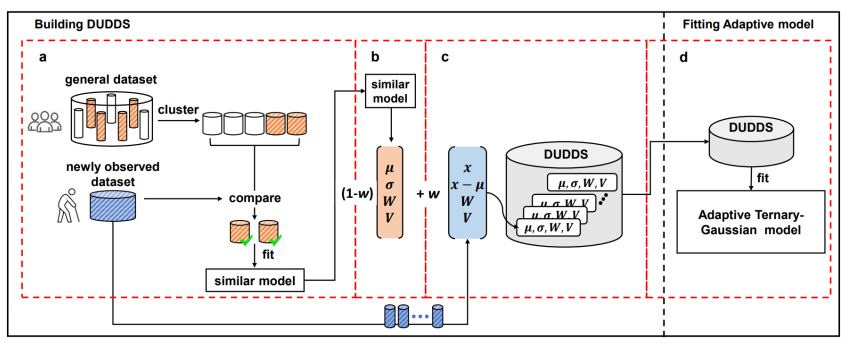

Figure 1: The process of modeling the Adaptive TernaryGaussian model.

In order to find a subset in the general dataset that is most similar to the newly observed dataset, we use the model coefficients of the 2D Ternary-Gaussian model to represent characterizes of each endpoint in the general dataset and select the subset based on these characterizes. K-means clustering on the general dataset's 
model coefficients is used to select the subset. After comparing the average model coefficient of the newly observed dataset with these clustering groups, the groups with the most similar characteristics of the newly observed dataset are filtered out. The endpoint data corresponding to the groups forms the subset, which is used to fit the similar model. Technical details of the similar model can be found in Appendix A.1.

Because the similar model is essentially a Ternary-Gaussian model, its output is the Gaussian parameters under the $W \times V$ conditions of the newly observed dataset. Therefore, we can use coordinates of the endpoints in the newly observed dataset to update the Gaussian parameters produced by the similar model. To update the Gaussian parameters produced by the similar model, we use a linear combination with weights to combine the Gaussian parameters and the coordinates of the endpoints in the newly observed dataset. We describe details of the linear combination method in Appendix A.2.

The weight of the linear combination $w$ can determine the tradeoff between the inherent characterizes and the newly observed characterizes when updating the Gaussian parameters. The larger the value of $w$, the more specific the characteristics of the newly observed data. $w$ is given by an SVM regression model. The SVM model aims to find the most appropriate update weight in the linear combination. See Appendix A.3 for more details of the SVM regression model.

In the following sections, we evaluate the performance of the adaptive Ternary-Gaussian model with two studies in specific users and pointing postures.

\section{STUDY 1: ADAPTATION OF THE MODEL TO USER CHARACTERISTICS}

In this section, we analyze and compare the differences in the endpoint distribution of three user groups, and evaluate the performance of our adaptive model in predicting error rates for different users. Besides, we explore the influence of motor ability on the user error rate of moving target selection.

\subsection{Participants and Apparatus}

We recruited 21 subjects (10 females and 11 males, with an average age of $35.0, S D=9.3$ ) as the general population, 12 older participants ( 4 females and 8 males, with an average age of $72.7, S D=4.3$ ) and 12 children ( 8 females and 4 males, with an average age of 4.4, $S D=0.6$ ) as the specific population with low motor ability. All participants were right-handed, and none of them had impaired motor ability.

Touch data was collected using a WeChat mini program developed by WeChat Developer Tools. All trials were run on an iPhone 6s Plus smartphone (5.5 inches diagonal with a screen resolution of $1920 \times 1080$ pixels) with a pixel density of 401ppi.

\subsection{Design and Procedure}

Each participant was asked to complete two tasks: a 2D Fitts' law experiment implemented according to the ISO 9241-9 standard [1] (Figure 2 (a)), and a moving target selection task (Figure 2 (a)). The 2D Fitts' law experiment was used to quantify human performance in target acquisition tasks. The moving target selection task is used to evaluate whether the adaptive model can work better than the general model. For these two tasks, participants were required to hold the device with one of their hands and tap the screen with the index finger of their dominant hand.

\subsubsection{Task 1: Fitts' law experiment.}

Our 2D Fitts' law experiment is designed according to the detailed description of the method provided by MacKenzie [24]. There are six combinations: $A=\{600,500,400\} p x \times W=\{200,140\} p x$. A combination is a sequence, including 20 trials. For each trial, participants were asked to point on a white circular target as quickly and accurately as possible. The participants could only point at the target once for each trial. They received hints by sound for hitting or missing the target. As shown in Figure 2, selections proceeded in a rotating pattern around a circle. We recorded endpoints data starting from the first selection until all 20 targets have been selected. Rest was allowed between each sequence. After the 6 sequences of the 2D Fitts' law experiment were completed, participants entered the moving target selection task.

\subsubsection{Task 2: Moving target selection.}

In each trial, participants were asked to point at a white moving circular target as quickly and accurately as possible. There were 4 levels of width $(W)$ and 4 levels of velocity $(V)$ of the targets:

$$
\begin{gathered}
W=\{90,120,150,180\} p x \\
V=\{72,132,192,252\} p x / s
\end{gathered}
$$

A total of 16 target conditions were included. Each target condition included 10 trials, yielding a total of 45 participants $\times 16$ conditions $\times 10$ trials $=7200$ taps. For each trial, we record the coordinates of the touch endpoint, the coordinates of the target, the target $\mathrm{W}, \mathrm{V}$, the direction of target movement, whether it is selected and the duration time it takes to acquire the target.

The trial started by the participant points to the stationary white circular target in the center of the screen. Once that trial started, a moving target appeared at a random location on the screen and moved at a fixed speed. Initial azimuth and moving direction were both randomized between 0 to $360^{\circ}$. The target would bounce back when its boundary touched the edge of the screen. We recorded endpoints data no matter the participant hit or miss the target. Notify sound for hitting or missing the target was provided. To prevent fatigue, participants had a 1-min break for every 40 taps.

\subsection{Measures}

4.3.1 Measure for user characteristics.

We used age and throughput (TP) to measure the characteristics of a user. By doing so, we can investigate the effects of users' characteristics on user error rates. TP can be calculated with the data collected in the Fitts' law experiment as follow,

$$
T P=\frac{I D_{e}}{M T}
$$

Where $I D_{e}$ is the effective index of difficulty of the task (in bits) calculated according to the movement amplitude and target width, and $\mathrm{MT}$ is the mean movement time (in seconds) recorded in a series of trials. The $I D_{e}$ term in equation (4) is expanded as follows:

$$
I D_{e}=\log _{2}\left(\frac{A_{e}}{W_{e}}+1\right)
$$


$A_{e}$ and $W_{e}$ in Equation (5) are the effective movement amplitude and effective width [24]. $A_{e}$ is the sum of the distance between two targets' centers a and $\mathrm{dx}$, with $\mathrm{dx}$ is the length of the projection of the user endpoint on the extension line of the target centers connection. With $W_{e}$ is computed as $4.133 \times S D_{x}$, where $S D_{x}$ is the standard deviation in the $d x$ values. Lower TP means the lower motor ability of the user.

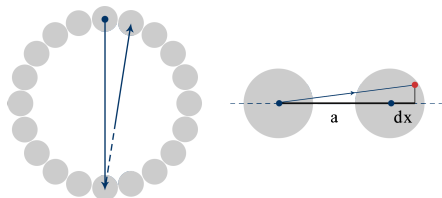

(a)

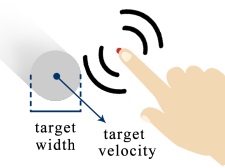

(b)
Figure 2: Task 1 (a) and task 2 (b) of Study 1 . The gray solid circle is the target, and its center is indicated by the blue dot. The red dot is the user actual endpoint position. As shown in (a), task 1 is Fitts' law serial 2D task.Users complete task 1 along the direction indicated by the blue arrow. The right half of (a) shows the geometry for a trial. As shown in (b), task 2 is a moving target pointing task. The blue arrow indicates the direction of the target moving. The target width is the diameter of the circle.

\subsubsection{Measure for user error rate.}

We collected the error rates of all trials in the moving target selection task. The error rate was calculated as the number of failed selections divide by the total number of selections. A failed selection was recorded as the Euclidean distance between the endpoint and the target center is greater than the target radius. We used the actual error rate as a measurement to evaluate user error rate.

4.3.3 Measure for model performance.

We used mean absolute error (MAE) between the model predicted error rate and the actual values as the measurement to evaluate the model performance.

\subsection{Results}

4.4.1 User Error Rate in Different User Groups.

In this section, we compare the differences in touch performance among three user groups and explore the influence of user characteristics on pointing performance using nonparametric correlation analysis.

A one-way ANOVA test showed a significant difference among the general group, the older group, and the child group ( $F=37.425$, $\mathrm{p}<.001)$. A Post-hoc test showed that there were significant differences between the general group and the older group $(\mathrm{p}<.001)$, the general group, and the child group $(\mathrm{p}<.001)$. The lowest average error rate was observed in the general group with $45.2 \%$, followed by $73.1 \%$ in the older group and $73.5 \%$ in the child group. The results indicated that compared with the general population, the older group and children group have a higher error rate.

Further, we conducted a Nonparametric correlation analysis between error rate and TP and age. As shown in Table 1, the correlation analysis of age is not obvious. We divided age into two
Table 1: Correlations between error rate and TP and age. "***” indicates correlations with significance levels above $99 \%$.

\begin{tabular}{ccc}
\hline Factor & TP & Age \\
\hline Correlation Coefficient & $-0.874^{* *}$ & 0.077 \\
Sig.(2-tailed) & $<0.001$ & 0.617 \\
\hline
\end{tabular}

levels for the analysis. The participants' data was divided into two segments with 33, the median age of the 45 participants. For participants younger than 33, a significant negative Spearman correlation coefficient $-0.759(\mathrm{p}<.001)$ between error rate and age was found. For participants older than 33, a significant positive coefficient of $0.623(\mathrm{p}=.002)$ between error rate and age was found. In addition, we found that the error rate has a significant negative correlation of $-0.874(\mathrm{p}<.001)$ with TP.

\subsubsection{Endpoint Distributions in Different User Groups.}

To understand the pointing uncertainty in moving target selection for the special population, we compare the actual endpoint distribution among the groups and report the estimated coefficients of the 2D Ternary-Gaussian model fitted by three groups respectively.

Figure 3 represents the actual endpoint distribution of the three groups and the 2 times standard deviation contours of predicted Ternary-Gaussian distributions when $\mathrm{W}=150 \mathrm{px}$ and $\mathrm{V}=252 \mathrm{px} / \mathrm{s}$. The results show that compared with the general people, the child and the older user group have higher uncertainty overall. In the $\mathrm{x}$-axis of the velocity coordinate system, the endpoints of the children and the older user group are more scattered, indicating larger pointing uncertainties for these two user groups. The shifting effect of endpoints, that is the effect of selection endpoints shift in the opposite direction of the target motion, for these two user groups are more obvious. In the y-axis, the pointing uncertainty of moving targets selection of the children and the older user group is remarkably higher compared to the general user group.
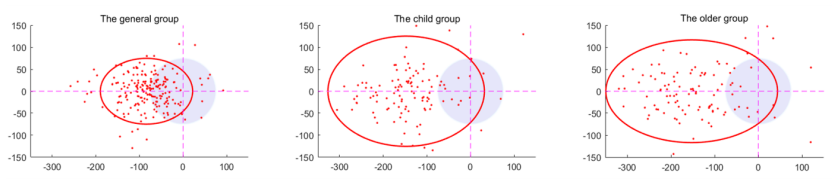

Figure 3: Actual endpoints and predicted distributions of $\mathrm{W}=150 \mathrm{px}$ and $\mathrm{V}=252 \mathrm{px} / \mathrm{s}$ condition of the three groups. The gray circles are the targets, and the red ellipses are 2 times standard deviation contours of predicted distributions. The red dots are actual endpoints.

Table 2 shows the coefficients of the Ternary-Gaussian model fitted by three groups respectively. $a_{x}, d_{x}$ and $d_{y}$ reflect absolute precision uncertainty. Belonging to the target velocity term, $b_{x}$, $e_{x}$ and $e_{y}$ can reflect the uncertainty effected by target speed. $c_{x}$, $f_{x}$ and $f_{y}$ are related to the precision tolerance of the target, $g_{x}$ is related to user speed-accuracy trade off.

Observing the coefficient of $\mu_{x}$, there are some findings related to the endpoint shifting effect. The $a_{x}$ of the general user group is larger and positive, indicating the general user group is more stable 
in touch performance and good at judging the location of the target in advance to tap on the target. The $b_{x}$ and $c_{x}$ of the general user group are smaller which indicates that their shifting effect caused by speed is smaller, and they are less reliant on the target size to compensate for the shifting effect. Correspondingly, the shifting effect of children and the older user group is largely affected by target speed, and they are more reliant on using the target size to compensate for the shifting effect.

The results also show some interesting findings of the uncertainty of the endpoint distribution. Among the correlation coefficients of $\sigma_{x}$, the smallest $d_{x}$ is the general population, indicating a smaller absolute uncertainty of the general population. The $d_{x}$ is the largest in the group of children, indicating a lower absolute accuracy for children. We attribute this as a typical Midas-Touch problem in children, that some of the touch behaviors in children are not intentional but unconscious and redundant behaviors. The $e_{x}$ of the general population is the smallest, indicating that the uncertainty of their endpoint distribution is less affected by speed. The $f_{x}$ of the three groups are close, which means that all users in the three groups utilize the same level of target width to compensate for the pointing uncertainty. The $d_{y}$ and $e_{y}$ of the general population are smaller than the other two populations, indicating that the absolute uncertainty of the general population in the y-axis is smaller and the distribution is denser. The $d_{y}$ of the older user group is the largest, indicating that the uncertainty of the older user group in the y-axis is the largest.

Table 3 shows the Spearman correlation coefficients of TP and all estimated coefficients. The results confirm the fact that TP has a significant correlation with $\mu_{x}$ and $\sigma_{x}$. The positive correlation coefficient between TP and $b_{x}$ is $0.736(\mathrm{p}<.001)$, which shows that the shifting effect of endpoints is greatly affected by the target speed. The negative correlation coefficients of TP and $d_{y}$ and $e_{y}$ are $-0.348(\mathrm{p}=.019)$ and $-0.326(\mathrm{p}=.029)$ respectively. $d_{y}$ increases with the increase of TP, that is, as the motor ability becomes lower, the pointing uncertainty will be higher.

\subsubsection{Model Evaluation.}

Model $_{\text {adp }}$, Model $l_{\text {genl }}$ and Model $_{\text {self }}$ respectively represent our adaptive 2D Ternary-Gaussian model, the general 2D TernaryGaussian model that pre-fitted by the general dataset, and the 2D Ternary-Gaussian model fitted by the newly observed dataset. Specifically, in Study 1, the general dataset contains the endpoints collected from the general population (21 adults with an average age of 35) and the newly observed dataset contains 20 endpoints from an individual of the specific populations (12 older participants with an average age of 72.7 and 12 children with an average age of 4.4).

Note that, for building the Adaptive Ternary-Gaussian model, we need to train an SVM regression model to determine the weight for updating the similar model. To construct the SVM regression model in Study 1, the data collection method described in Appendix A.3 was repeated 5720 times. Finally, the training dataset contains 5720 records. The trained SVM model got a fit of $0.599 R^{2}$ in the data. Although the model did not get high performance for predicting the ideal weight for updating the similar model, we will see later that the overall performance of our final Adaptive Ternary-Gaussian model is still better than the baseline.
We used the MAE of the predicted error rate of the model as a measurement to evaluate the model performance. Specifically, we randomly selected 20 endpoints from the endpoints collected from each participant in the experiment as the newly observed dataset. We computed the MAE after building our adaptive TernaryGaussian model using the selected 20 endpoints. We then repeated the process described above 100 times to obtain a stable MAE for one participant. Finally, the average MAE of all participants in the group was used to evaluate our model.

Since the MAE of the predicted error rate of models was not normally distributed, we used the non-parametric Kruskal-Wallis test [39] for the statistical test. The results of the analysis are shown in figure 4.
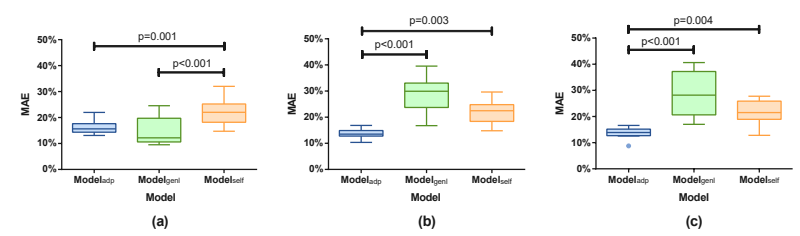

Figure 4: The MAE of predicted error rate of the three models in the general group, the child group, and older group (from left to right).

For the general group, the differences among MAE of the three models were significant $(\mathrm{H}=22.475, \mathrm{P}<.001)$. Post-hoc tests showed Model $_{a d p}$ and Model $_{\text {genl }}$ performed significantly better than Model $_{\text {self }}$ (both $\mathrm{p} \leq .001$ ). No significant difference was found between Model $_{a d p}$ and Model $_{\text {genl }}$. Among the three models, the lowest average of MAE of the predicted error rate is Model $_{\text {genl }}$ at $14.8 \%$, followed by Model $_{a d p}$ at $16.4 \%$ and Model $_{\text {self }}$ at $21.9 \%$. The results indicate that for the general group our adaptive modeling have similar performance as Model $_{\text {genl }}$ while it perform significantly than Model $_{\text {self }}$.

For the child group, the differences among MAE of the three models were significant $(\mathrm{H}=24.721, \mathrm{p}<.001)$. Post-hoc tests showed Model $_{a d p}$ performed significantly better than both Model $_{\text {genl }}$ and Model $_{\text {self }}$ (both $\left.\mathrm{p}<.001\right)$. No significant difference was found in MAE between Model $_{\text {genl }}$ and Model $_{\text {self }}(\mathrm{p}=.311)$. Among the three models, the lowest average MAE of the error rate of $13.5 \%$ was observed at Model $_{a d p}$, followed by Model $_{\text {self }}$ at $22.2 \%$ and Model $_{\text {genl }}$ at $28.7 \%$. The results indicate that our adaptive model can significantly improve the predicted accuracy when applied to the child.

For the older user group, the differences among MAE of the three models were significant $(\mathrm{H}=22.416, \mathrm{p}<.001)$. Post-hoc tests showed Model $_{\text {adp }}$ performed significantly better than both Model $_{\text {genl }}(\mathrm{p}<$ $.001)$ and Model $_{\text {self }}(\mathrm{p}=.004)$. No significant difference was found in MAE between Model $_{\text {genl }}$ and Model self $(\mathrm{p}=.507)$. Among the three models, the lowest average MAE of the error rate of $13.7 \%$ was observed at Model $_{a d p}$, followed by Model $_{\text {self }}$ of $21.5 \%$ and Model $_{\text {genl }}$ of $28.5 \%$. The results indicate that our adaptive model can significantly improve the predicted accuracy when applied to the older user.

In Figure 5 (a), we plot the MAE of Model $_{a d p}$ and Model genl along with users pointing error rates as functions of TP. Each 
Table 2: Estimated coefficients of the Ternary-Gaussian model fitted respectively by three groups' endpoints.

\begin{tabular}{cccccc}
\hline & Coefficient & Term & The general group & The child group & The older group \\
\hline$\mu_{x}$ & $a_{x}$ & - & 16.3636 & -0.5522 & 6.7006 \\
& $b_{x}$ & $V$ & -0.4015 & -0.6222 & -0.6509 \\
& $c_{x}$ & $W$ & 0.0057 & 0.0652 & 0.0329 \\
$\sigma_{x}$ & $d_{x}$ & - & 749.2142 & 1589.1499 & 1133.9425 \\
& $e_{x}$ & $V^{2}$ & 0.0243 & 0.0924 & 0.1271 \\
& $f_{x}$ & $W^{2}$ & 0.0225 & 0.0258 & 0.0163 \\
$\sigma_{y}$ & $g_{x}$ & $V / W$ & 0.0008 & 0.0000 & 41.8169 \\
& $d_{y}$ & - & 810.7884 & 1593.5371 & 2127.7088 \\
& $e_{y}$ & $V^{2}$ & 0.0054 & 0.0321 & 0.0201 \\
& $f_{y}$ & $W^{2}$ & 0.0112 & 0.0139 & 0.0000 \\
\hline
\end{tabular}

Table 3: Correlation analysis between TP and estimated coefficients. "**" indicates correlations with significance levels above 99\%. "*" indicates correlations with significance levels above $95 \%$.

\begin{tabular}{ccccccccccc}
\hline & \multicolumn{3}{c}{$\mu_{x}$} & \multicolumn{1}{c}{$\sigma_{x}$} & \multicolumn{9}{c}{$\sigma_{y}$} \\
\hline Factor & $a_{x}$ & $b_{x}$ & $c_{x}$ & $d_{x}$ & $e_{x}$ & $f_{x}$ & $g_{x}$ & $d_{y}$ & $e_{y}$ & $f_{y}$ \\
Correlation Coefficient & $0.374^{*}$ & $0.736^{* *}$ & $-0.298^{*}$ & 0.063 & -0.262 & 0.095 & -0.151 & $-0.348^{*}$ & $-0.326^{*}$ & $-0.319^{*}$ \\
Sig.(2-tailed) & 0.011 & $<0.001$ & 0.047 & 0.683 & 0.082 & 0.534 & 0.322 & 0.019 & 0.029 & 0.032 \\
\hline
\end{tabular}

point in the figure represents a user. We can see clearly from the figure that users pointing error rate increases as TP decreases; the performance of Model $_{\text {genl }}$ becomes wore as TP decreases, while our adaptive model Model $_{a d p}$ maintains good performance.

In Figure 5 (b), we plot the MAE of Model $_{a d p}$ and Model ${ }_{\text {genl }}$ along with users pointing error rates as functions of age. Each point in the figure represents a user. The figure reveals that users in younger and older age groups have a worse pointing accuracy; The general model Model $_{\text {genl }}$ can performs well within age group of 20-50 years old, while its performance decline rapidly in age groups lower than 10 years old and higher than 70 years old. On the contrary, our adaptive model Model $_{a d p}$ maintains good performance across all age groups.

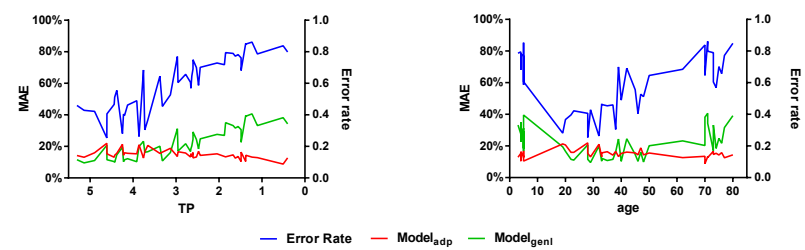

Figure 5: The MAE of the predicted error rate of the Model $_{a d p}$ and Model $_{\text {genl }}$ varies with TP and age.

\subsection{Discussion}

We compared the endpoint distribution of three groups and the coefficients of the Ternary-Gaussian model of three groups. The results show that the general group's shifting effect is smaller, and the overall uncertainty is lower. The endpoints of the two specific user groups are severely shifting and scattered. The endpoints of the two specific user groups are greatly affected by target speed.

The results of the model evaluation show that our adaptive model is significantly helpful for users with low motor ability. For the older user group and children, Model $_{a d p}$ performed significantly better than Model $_{\text {genl }}$, increasing by $15.1 \%$ and $14.8 \%$, respectively. For the general population, there is no significant difference in the performance of Model $_{a d p}$ and Model $_{\text {genl }}$. Users with high motor ability have high accuracy, most of the endpoints fall within the target, and the endpoint distribution is more related to the target width. At this time, Model $_{a d p}$ will have the same performance as Model $_{\text {genl }}$. However, users with low motor ability have a distribution that is very different from the general people. At this time, our adaptive model plays an advantage.

As TP decreasing, the error rate of participants increases. The adaptive model can adapt to this change and keep the predicted error rate MAE stable. The MAE of Model $_{\text {genl }}$ predicted error rate does not adapt to user characteristics well, increasing while user TP decreasing. We can see that for users with worse performance, Model $_{a d p}$ can predict the error rate of moving target selection more accurately than Model $_{\text {genl }}$.

\section{STUDY 2: ADAPTATION OF THE MODEL TO POINTING POSTURES}

To evaluate our model in different pointing postures, we designed and implemented Study 2. We analysed the predicted accuracy of Model $_{a d p}$, Model $_{\text {genl }}$ and Model $_{\text {self }}$ under 4 postures. Furthermore, we explored the changes in user pointing error rate due to status restrictions. 


\subsection{Participants and Apparatus}

We recruited 14 subjects ( 8 females and 6 males, with an average age of 28.0, $S D=4.0$ ). All participants were right-handed, and none of them had gait-related issues. The same as Study 1 , an iPhone $6 \mathrm{~s}$ Plus smartphone was used to conduct the experiment.

\subsubsection{Design and Procedure.}

Study 2 is designed to be implemented under the four postures (shown as Figure 6) as follow:

- Posture 1 (P1): Sitting at a table, hold the smartphone with the non-dominant hand and select the targets with the index finger of the dominant hand.

- Posture 2 (P2): Sitting at a table, hold the smartphone with the dominant hand and select the targets with the thumb of the dominant hand.

- Posture 3 (P3): Walking, hold the smartphone with the nondominant hand, and select the targets with the index finger of the dominant hand.

- Posture 4 (P4): Walking, hold the smartphone with the dominant hand and select the targets with the thumb of the dominant hand.

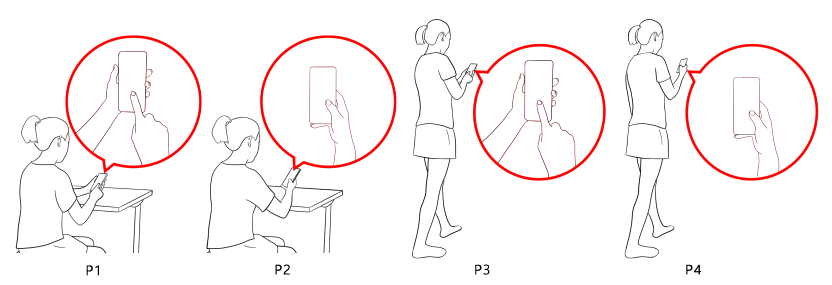

Figure 6: Diagram of four postures in Study 2. P1-4 from left to right.

Participants were asked to walk in a straight line in a room. The order of the postures follows the Latin square. Participants are asked to tap on the moving white circular target which appeared on the screen randomly. Each of the 16 combinations of $\mathrm{W}$ and $\mathrm{V}$ appeared 10 times. Each condition includes 160 trials, a total of 14 participants $\times 4$ status $\times 16$ conditions $\times 10$ trials $=8960$ taps were recorded.

\subsection{Measures}

Same as Study 1, we use the MAE of the model predicted error rate as a measurement to evaluate the model performance, and we use the user actual pointing error rate as a measurement to evaluate the user error rate.

\subsection{Results}

\subsubsection{User Error Rate in Different Postures.}

We used a one-way ANOVA test to analyze the user error rate of the pointing moving target among four postures. A significant difference was found among P1-4 $(\mathrm{F}=7.106, \mathrm{p}<.001)$.

Post-hoc test showed that there were significant differences between P1 and P2 (p= .002), P1 and P3 (p= .003), P1 and P4 (p< .001 ). The lowest average error rate was observed in $\mathrm{P} 1$ with $37.3 \%$, followed by $51.3 \%$ of P3 and $52.0 \%$ of P2. P4 has the highest error rate of 57.3\%. Compared with sitting at a table, walking has lower accuracy of pointing at moving targets. For the condition of sitting at a table, one-hand had a higher error rate than two-handed.

\subsubsection{Endpoint Distribution in Different Postures.}

To understand the uncertainty of pointing at moving targets in specific status, we compare the actual endpoint distribution of four postures of using a smartphone and report the estimated coefficients of the Ternary-Gaussian model fitted by endpoint data in P1-4 respectively.

Figure 7 represents the actual endpoint distribution of the four postures and the 2 times standard deviation contours of predicted Ternary-Gaussian distributions when $\mathrm{W}=150 \mathrm{px}$ and $\mathrm{V}=252 \mathrm{px} / \mathrm{s}$. Compared with P1, P2-4 has higher overall uncertainty. P2-4 has a greater shifting effect in the $\mathrm{X}$-axis of the velocity coordinate system. The endpoint distribution of $\mathrm{P} 3$ is more affected by velocity. In the y-axis, endpoints of P2-4 are more scattered, accompanied by greater deviation. When walking or using a smartphone with one hand, the user's pointing uncertainty is higher.
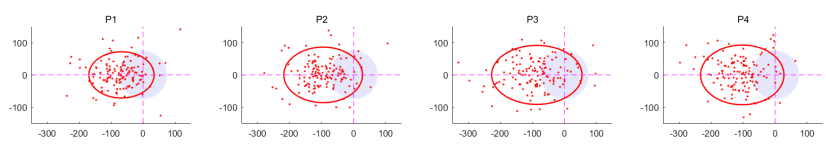

Figure 7: Actual endpoints and predicted Ternary-Gaussian distributions of $\mathrm{W}=150 \mathrm{px}$ and $\mathrm{V}=252 \mathrm{px} / \mathrm{s}$ condition under four postures. The gray circles are the targets, and the red ellipses are 2 times standard deviation contours of predicted distributions. The red dots are actual endpoints.

Table 4 shows the coefficients of the Ternary-Gaussian model fitted by four postures respectively. Observing the coefficient of $\mu_{x}$, there are some findings related to the endpoint shifting effect. The $a_{x}$ of $\mathrm{P} 3$ is larger and positive, indicating that users in P3 are good at judging the location of the target in advance. Their $c_{x}$ are smaller which indicates that they are less dependent on the target size to compensate shifting effect. The $b_{x}$ of $\mathrm{P} 1$ is smaller, which means the users shifting effect caused by target speed is smaller in P1. Correspondingly, the shifting effect of P2-4 is largely affected by speed. Among the correlation coefficients of $\sigma_{x}$, we found that $d_{x}$ of $\mathrm{P} 1$ and $\mathrm{P} 2$ is smaller, indicating that users' absolute uncertainty in $\mathrm{P} 1$ and $\mathrm{P} 2$ is smaller because the sitting posture has higher stability. The $e_{x}$ of $\mathrm{P} 1$ is the smallest, indicating that the endpoint distribution uncertainty is less affected by the target speed in P1. Correspondingly, the $e_{x}$ of $\mathrm{P} 3$ is the largest, indicating that target speed especially has an impact on endpoint distribution. P3 and $\mathrm{P} 4$ have larger $f_{x}$, which means that $\mathrm{P} 3$ and $\mathrm{P} 4$ rely more on the width of the target to compensate for the uncertainty. The $d_{y}$ and $e_{y}$ of $\mathrm{P} 1$ are smaller than the other three postures, indicating that the absolute uncertainty in the $y$-axis is smaller and the distribution is denser when the user points moving target with two hands while sitting. The $d_{y}$ of $\mathrm{P} 3$ is the largest, indicating that the uncertainty of $\mathrm{P} 3$ in the $\mathrm{y}$-axis is the largest.

\subsubsection{Model Evaluation.}

In Study 2, the general dataset which fits the Model $_{\text {genl }}$ contains the all endpoints collected in general usage scenario (P1), and the newly 
Table 4: Estimated coefficients of the Ternary-Gaussian model fitted by four postures respectively.

\begin{tabular}{ccccccc}
\hline & Coefficient & Term & P1 & P2 & P3 & P4 \\
\hline \multirow{4}{*}{$\mu_{x}$} & $a_{x}$ & - & 9.2392 & 13.9396 & 23.9378 & 11.1766 \\
& $b_{x}$ & $V$ & -0.3326 & -0.4760 & -0.4265 & -0.4981 \\
\multirow{6}{*}{$\sigma_{x}$} & $c_{x}$ & $W$ & 0.0475 & 0.0728 & 0.0155 & 0.0786 \\
& $d_{x}$ & - & 618.7832 & 1155.7505 & 958.5001 & 1282.9767 \\
& $e_{x}$ & $V^{2}$ & 0.0280 & 0.0365 & 0.0457 & 0.0331 \\
& $f_{x}$ & $W^{2}$ & 0.0093 & 0.0132 & 0.0447 & 0.0379 \\
$\sigma_{y}$ & $g_{x}$ & $V / W$ & 0.1099 & 0.0006 & 64.8547 & 0.0522 \\
& $d_{y}$ & - & 712.1131 & 843.5597 & 1170.3732 & 1118.9169 \\
& $e_{y}$ & $V^{2}$ & 0.0054 & 0.0074 & 0.0079 & 0.0072 \\
& $f_{y}$ & $W^{2}$ & 0.0103 & 0.0236 & 0.0181 & 0.0247 \\
\hline
\end{tabular}

observed dataset which fits the Model $_{\text {self }}$ contains 20 endpoints collected from an individual in specific usage scenarios (P2, P3, $\mathrm{P} 4)$. The endpoints in the general dataset and the newly observed dataset train the Model $_{a d p}$ for that specific user.

As stated before, we use the MAE of the predicted error rate to evaluate our model. Again, the MAE of the predicted error rate of models was not normally distributed, so we used the nonparametric Kruskal-Wallis test to analyze the data.

For P1, the differences among MAE of the three models were significant $(\mathrm{H}=30.490, \mathrm{P}<.001)$. Post-hoc tests showed Model $_{a d p}$ and Model $_{\text {genl }}$ performed significantly better than Model $_{\text {self }}$ (both $\mathrm{p}<$ .001). No significant difference was found between Model $_{a d p}$ and

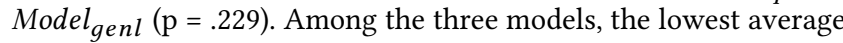
MAE of the predicted error rate is Model $_{\text {genl }}$ at $13.0 \%$, followed by Model $_{a d p}$ at $16.1 \%$ and Model $_{\text {self }}$ at $23.7 \%$. The results indicate that for the P1 our adaptive modeling have similar performance as Model $_{\text {genl }}$ while it perform significantly better than Model $_{\text {self }}$ by reducing $10.7 \% \mathrm{MAE}$.

For P2, the differences among MAE of the three models were significant $(\mathrm{H}=29.548, \mathrm{P}<.001)$. Post-hoc tests showed Model $_{a d p}$ performed significantly better than Model $_{\text {genl }}$ and Model self $_{\text {(both }}$ $\mathrm{p}<.001)$. No significant difference was found between Model $_{g e n l}$ and Model $_{\text {self }}(\mathrm{p}=.417)$. Among the three models, the lowest average MAE of the predicted error rate is Model $_{a d p}$ at $14.4 \%$, followed by Model $_{\text {genl }}$ at $19.4 \%$ and Model $_{\text {self }}$ at $22.4 \%$. Our model had $5.0 \%$ and $9.0 \%$ lower average MAEs compared to the general model Model $_{\text {genl }}$ and Model $_{\text {self }}$, respectively.

For P3, the differences among MAE of the three models were significant $(\mathrm{H}=23.710, \mathrm{P}<.001)$. Post-hoc tests showed Model $_{\text {ad }}$ performed significantly better than Model $_{\text {genl }}$ and Model $_{\text {self }}$ (both $\mathrm{p} \leq .001)$. No significant difference was found between Model $_{\text {genl }}$ and Model $_{\text {self }}(\mathrm{p}=1.000)$. Among the three models, the lowest average MAE of the predicted error rate is Model $_{a d p}$ at $14.4 \%$, followed by Model $_{\text {genl }}$ at $24.0 \%$ and Model $_{\text {self }}$ at $26.4 \%$. Our model had $9.5 \%$ and $12.0 \%$ lower average MAEs compared to the general model Model ${ }_{\text {genl }}$ and Model $_{\text {self }}$, respectively.

For $\mathrm{P} 4$, the differences among MAE of the three models were significant $(\mathrm{H}=28.504, \mathrm{P}<.001)$. Post-hoc tests showed Model $_{\text {adp }}$ performed significantly better than Model $_{\text {genl }}$ and Model $_{\text {self }}$ (both $\mathrm{p} \leq .002$ ). No significant difference was found between Model $_{\text {genl }}$ and Model $l_{\text {self }}(\mathrm{p}=.160)$. Among the three models, the lowest average MAE of the predicted error rate is Model $_{a d p}$ at $13.9 \%$, followed by Model $_{\text {genl }}$ at $21.4 \%$ and Model $_{\text {self }}$ at $27.1 \%$. Our model had a $13.2 \%$ and $7.5 \%$ lower average MAEs compared to the general model Model $_{\text {genl }}$ and Model $_{\text {self }}$, respectively.

In summary, compared to the general model Model genl $_{\text {, our }}$ adaptive model Model $_{a d p}$ performed significantly better in P2-P4; compared to the model fitted by the newly observed data Model $_{\text {self }}$, our adaptive model Model $_{a d p}$ performed significantly better in all the four postures.

It can be seen from the results of Study 1 that differences in user error rate will lead to changes in model performance. As shown in Figure 8, we observe that our adaptive model Model $_{a d p}$ has maintained a stable accuracy rate prediction performance, while the performance of Model $_{\text {genl }}$ and Model $_{\text {self }}$ deteriorated. This confirms that differences caused by use scenarios can also be adapted by Model $_{a d p}$.

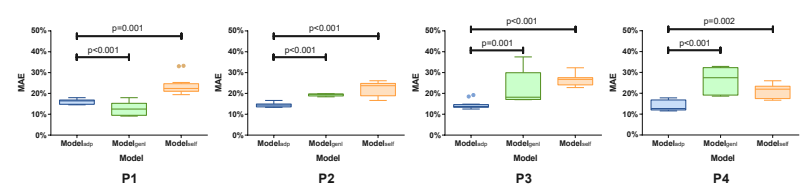

Figure 8: The MAE of the predicted error rate of three models under four postures.

\subsection{Discussion}

The Adaptive Ternary-Gaussian model works well in different postures while the general model performs badly. This shows that the model is robust and adaptable to differences caused by situational impairments.

Different usage posture has a significant impact on the user error rate in moving target selection task. Specifically, walking has more negative effects on selection accuracy than sitting, which is consistent with the conclusions of previous studies [5]. The results show that the endpoint distribution has higher absolute uncertainty and is more affected by target speed when pointing at moving targets with both hands while walking. At the same time, the endpoint distribution uncertainty of pointing is minimal with both hands while 
sitting. When walking, the shifting effect of endpoint distribution is more obvious of two-handed than one-handed which may be because the cooperation of the two hands brings more uncertainty.

\section{GENERAL DISCUSSION}

\subsection{Model Performance}

We used mean absolute error (MAE) between predicted error rates and actual error rates to evaluate our model. In Study 1, the MAE of the predicted error rates of the adaptive model for children and older participants are $13.5 \%$ and $13.7 \%$, respectively. Our model had $15.2 \%$ and $14.8 \%$ lower average MAEs compared to the general model. In Study 2, the MAE of the predicted error rates of the adaptive model for the three target pointing postures (i.e., P2, P3, and $\mathrm{P} 4$ ) are $14.4 \%, 14.4 \%, 13.9 \%$, respectively. Our model had $5.0 \%$, $9.5 \%$ and $13.2 \%$ lower average MAEs compared to the baseline. These results demonstrate that our proposed model can quickly learn the variations in endpoints created by the above two specific circumstances. Further, when faced with varying circumstances, the model is stable, regardless of whether the discrepancy is caused by the user's physiological features or situational obstacles.

Comparing our results to previous Ternary-Gaussian model experiments [15-17], the overall model performances are much lower (about 15\% MAE) than the previous ones (<5\% MAE). We attribute this is to the variability introduced by different user groups and pointing postures. In the previous works, the models were trained and evaluated within the same user group and posture. However, in the present studies, we adapt our model initially obtained from the general dataset to the target scenarios by absorbing information from a small amount of data (20 endpoints) from the target scenarios. This introduces more uncertainties comparing the previous works.

\subsection{Findings}

Our results show that the low motor ability of specific users may lead to a decline in pointing accuracy. Compared with the endpoint distribution of the general group, the older user group showed a larger shifting effect while the children have higher absolute uncertainties. The pointing performance of the older user group is greatly affected by target speed. One of the reasons is their reactions are generally slow. The unconscious error touch produced by the child makes their absolute accuracy lower.

In addition to motor ability, we found that situational obstacles can also cause uncertainty and affect pointing accuracy. Compared with stable sitting, walking brings more uncertainty of pointing at moving targets. While walking, One-handed posture introduced higher uncertainty in the endpoint distribution than the cooperative use of both hands. Compared with sitting $\times$ two-handed, walking and one-handed introduced higher uncertainty in the endpoint distribution. Besides, one-handed has a higher absolute uncertainty while the two-handed has a greater shifting effect on endpoints.

Comparing the model coefficients of Study 2 with Study 1, we found two interesting findings of the disparities in the deviations introduced by user age groups and pointing postures: On one hand, the $b_{x}$ of age groups in Study 1 exhibits greater variations (ranging from -0.4015 to -0.6509 ) than the pointing posture groups (ranging from -0.3326 to -0.4981 ) in Study 2. This suggests that target speed could have a larger impact on children and older users, while the influences of target speed are relatively lower when users change their pointing posture. On the other hand, observing the coefficients of $\sigma_{y}, d_{y}$ and $e_{y}$ of the adaptive model of children $\left(d_{y}=1593.5371\right.$, $\left.e_{y}=0.0321\right)$ and the older group $\left(d_{y}=2127.7088, e_{y}=0.0201\right)$ are much larger than the general group $\left(d_{y}=810.7884, e_{y}=0.0054\right)$ while there is no apparent difference of the $d_{y}$ and $e_{y}$ among the four pointing postures $\left(d_{y}\right.$ ranged from 712.1131 to 1170.3732 , and $e_{y}$ ranged from 0.0054 to 0.0079 ) in Study 2. This demonstrates that the accuracy in the y-axis is more affected by age compared to pointing postures.

\subsection{Applying the Model in Practices}

Our solution imposes no extra technical pressure on consumers by using the proposed model in practice. The model dynamically captures user data and adjusts to the user's preferences. Any time the user tries to select a target, an endpoint is added to the newly observed dataset. Once the newly observed dataset has more than a sufficient number of endpoints (e.g., 20), the system will reconstruct the adaptive model on a regular basis to keep it fit for the most recently observed user data.

In the reconstructing process, the most time-consuming parts are clustering the general dataset using the K-means algorithm and training the SVM model. Fortunately, both of these two steps can be done offline. What needs to be done in real-time is to dynamically update the DUDDS and conducted the final model fitting with the Gaussian parameters in the DUDDS. In our offline testing environment (i.e., Matlab running in a commercial laptop computer), the above process takes about 1 second to complete. Moreover, the frequency of reconstructing the model can be adjusted according to the device's performance. For example, to minimize the calculation amount, the rebuild can be called once every 10 endpoints are obtained.

In real-world applications, our adaptive model can benefit older smartphone users. For example, by combining our model with the previous 2D-BayesPointer [17], an older person could acquire targets on a touchscreen more precisely, despite the fact that their pointing behavior differed substantially from that of the general public.

\subsection{Limitation and Future Work}

There are three limitations in this work. First, we did test the performance of our model in adapting many other important factors such device and screen size that have potentials influences on touchscreen pointing. Second, we only compared our model with the original 2D Ternary-Gaussian model, but not with other latest adaptive models such as the Cluster Touch [29]. A study comparing different adaptive models in the future could be beneficial. Another limitation of our work is that we test relatively small ranges of target initial distance, size and speed in the two studies. These task conditions could be selected more strategically to allow for a wider ID range.

In the future, we are interested in using our model to infer the characteristics of the users or the environments. For example, since our model can describe the difference on user pointing uncertainty in different user groups, it could be interesting to see whether we 
can use the model coefficients to assess the user's age by a few taps of the user on a touchscreen. Furthermore, we want to broaden and expand our research to other interaction modalities, such as AR/VR or tangible user interface, which may offer insights for a broader spectrum of interaction mechanisms.

\section{CONCLUSION}

This paper presents an adaptive model that can quickly learn the characterizes of endpoints from small data in a specific scenario. We evaluate the proposed model in specific users of children and the older people, and in specific pointing postures for walking and one-handed with a smartphone. The model is built by establishing a dynamically updated distribution dataset, which is used to fit the final 2D Ternary-Gaussian model adaptive to the specific scenarios. In both experiments, the adaptive model maintains good performances in predicting error rates for the specific user circumstances. Our adaptive model has a significantly lower MAE of predicted pointing error rate than the general model, indicating the robustness and generalization of the model.

\section{ACKNOWLEDGMENTS}

This work was supported by National Natural Science Foundation of China (Grant Nos. 61802379, 61802232, 62007021), Youth Innovation Promotion Association CAS (Grant No. 2020113), and Young Scholars Program of Shandong University, Weihai (20820211005).

\section{REFERENCES}

[1] 2000. Ergonomic requirements for office work with visual display terminals (VDTs) - Part 9: Requirements for non-keyboard input devices(ISO 9241-9). (2000).

[2] Zahouani Abdouni, Vargiolu. 2018. Impact of finger biophysical properties on touch gestures and tactile perception: Aging and gender effects. Scientific reports (2018). https://doi.org/10.1038/s41598-018-30677-2

[3] Abir Al Hajri, Sidney Fels, Gregor Miller, and Michael Ilich. 2011. Moving target selection in 2D graphical user interfaces. In IFIP Conference on Human-Computer Interaction. Springer, 141-161.

[4] L. Anthony, Q. Brown, J. Nias, B. Tate, and S. Mohan. 2012. Interaction and Recognition Challenges in Interpreting Children's Touch and Gesture Input on Mobile Devices. In Proceedings of the ACM International Conference on Interactive Tabletops and Surfaces.

[5] Joanna Bergstrom-Lehtovirta, Antti Oulasvirta, and Stephen Brewster. 2011 The Effects of Walking Speed on Target Acquisition on a Touchscreen Interface. In Proceedings of the 13th International Conference on Human Computer Interaction with Mobile Devices and Services (Stockholm, Sweden) (MobileHCI '11). Association for Computing Machinery, New York, NY, USA, 143-146. https://doi.org/10.1145/2037373.2037396

[6] Xiaojun Bi and Shumin Zhai. 2016. Predicting Finger-Touch Accuracy Based on the Dual Gaussian Distribution Model. In Proceedings of the 29th Annual Symposium on User Interface Software and Technology (Tokyo, Japan) (UIST '16). Association for Computing Machinery, New York, NY, USA, 313-319. https: //doi.org/10.1145/2984511.2984546

[7] Pradipta Biswas and Patrick Langdon. 2012. Developing Multimodal Adaptation Algorithm for Mobility Impaired Users by Evaluating Their Hand Strength. International fournal of Humancomputer Interaction 28, 9 (2012), 576-596.

[8] Daniel Buschek, Simon Rogers, and Roderick Murraysmith. 2013. User-specific touch models in a cross-device context. (2013), 382-391.

[9] Rachel Eardley, Anne Roudaut, Steve Gill, and Stephen J. Thompson. 2018. Investigating How Smartphone Movement is Affected by Body Posture. In Proceedings of the 2018 CHI Conference on Human Factors in Computing Systems (Montreal QC, Canada) (CHI '18). Association for Computing Machinery, New York, NY, USA, 1-8. https://doi.org/10.1145/3173574.3173776

[10] Michal Elboim Gabyzon, Lorenzo Chiari, Shlomi Laufer, Mattia Corzani, and Alexandra Danial-Saad. 2019. Evaluation of Touch Technology for the Aging Population. In 2019 International Conference on Virtual Rehabilitation (ICVR). 1-6. https://doi.org/10.1109/ICVR46560.2019.8994539

[11] Mayank Goel, Leah Findlater, and Jacob Wobbrock. 2012. WalkType: Using Accelerometer Data to Accomodate Situational Impairments in Mobile Touch Screen Text Entry. In Proceedings of the SIGCHI Conference on Human Factors in
Computing Systems (Austin, Texas, USA) (CHI '12). Association for Computing Machinery, New York, NY, USA, 2687-2696. https://doi.org/10.1145/2207676. 2208662

[12] J. A. Hartigan and M. A. Wong. 1979. A K-Means Clustering Algorithm. Applied Stats 28, 1 (1979). https://doi.org/10.2307/2346830

[13] Khalad Hasan, Tovi Grossman, and Pourang Irani. 2011. Comet and Target Ghost: Techniques for Selecting Moving Targets. In Proceedings of the SIGCHI Conference on Human Factors in Computing Systems (Vancouver, BC, Canada) (CHI '11). Association for Computing Machinery, New York, NY, USA, 839-848. https://doi.org/10.1145/1978942.1979065

[14] HOFFMANN and R. Errol. 1991. Capture of moving targets : a modification of Fitts' Law. Ergonomics 34, 2 (1991), 211-220. http://dx.doi.org/10.1080/ 00140139108967307

[15] Jin Huang, Feng Tian, Xiangmin Fan, Huawei Tu, Hao Zhang, Xiaolan Peng, and Hongan Wang. 2020. Modeling the Endpoint Uncertainty in Crossing-Based Moving Target Selection. In Proceedings of the 2020 CHI Conference on Human Factors in Computing Systems (Honolulu, HI, USA) (CHI '20). Association for Computing Machinery, New York, NY, USA, 1-12. https://doi.org/10.1145/ 3313831.3376336

[16] Jin Huang, Feng Tian, Xiangmin Fan, Xiaolong (Luke) Zhang, and Shumin Zhai. 2018. Understanding the Uncertainty in 1D Unidirectional Moving Target Selection. In Proceedings of the 2018 CHI Conference on Human Factors in Computing Systems (Montreal QC, Canada) (CHI '18). Association for Computing Machinery, New York, NY, USA, 1-12. https://doi.org/10.1145/3173574.3173811

[17] Jin Huang, Feng Tian, Nianlong Li, and Xiangmin Fan. 2019. Modeling the Uncertainty in 2D Moving Target Selection. In Proceedings of the 32nd Annual ACM Symposium on User Interface Software and Technology (New Orleans, LA, USA) (UIST '19). Association for Computing Machinery, New York, NY, USA, 1031-1043. https://doi.org/10.1145/3332165.3347880

[18] Curt B. Irwin and Mary E. Sesto. 2012. Performance and touch characteristics of disabled and non-disabled participants during a reciprocal tapping task using touch screen technology. Applied Ergonomics 43, 6 (2012), 1038-1043.

[19] R. J. Jagacinski, D. W. Repperger, S. L. Ward, and M. S. Moran. 1980. A test of Fitts' law with moving targets. Human Factors 22, 2 (1980), 225.

[20] Shaun K. Kane, Jacob O. Wobbrock, and Ian E. Smith. 2008. Getting off the Treadmill: Evaluating Walking User Interfaces for Mobile Devices in Public Spaces. In Proceedings of the 10th International Conference on Human Computer Interaction with Mobile Devices and Services (Amsterdam, The Netherlands) (MobileHCI '08). Association for Computing Machinery, New York, NY, USA, 109-118. https: //doi.org/10.1145/1409240.1409253

[21] Byungjoo Lee, Sunjun Kim, Antti Oulasvirta, Jong-In Lee, and Eunji Park. 2018. Moving Target Selection: A Cue Integration Model. In Proceedings of the 2018 CHI Conference on Human Factors in Computing Systems (Montreal QC, Canada) (CHI '18). Association for Computing Machinery, New York, NY, USA, 1-12. https://doi.org/10.1145/3173574.3173804

[22] Byungjoo Lee and Antti Oulasvirta. 2016. Modelling error rates in temporal pointing. In Proceedings of the 2016 CHI Conference on Human Factors in Computing Systems. 1857-1868.

[23] Min Lin, Rich Goldman, Kathleen J. Price, Andrew Sears, and Julie Jacko. 2007. How Do People Tap When Walking? An Empirical Investigation of Nomadic Data Entry. Int. 7. Hum.-Comput. Stud. 65, 9 (Sept. 2007), 759-769. https://doi. org/10.1016/j.ijhcs.2007.04.001

[24] I. Scott Mackenzie. 2015. Fitts' Throughput and the Remarkable Case of TouchBased Target Selection. In International Conference on Human-computer Interaction. https://doi.org/10.1007/978-3-319-20916-6_23

[25] Sven Mayer, Huy Viet Le, Alessandro Nesti, Niels Henze, Heinrich H. Bülthoff, and Lewis L. Chuang. 2018. The Effect of Road Bumps on Touch Interaction in Cars. In Proceedings of the 10th International Conference on Automotive User Interfaces and Interactive Vehicular Applications (Toronto, ON, Canada) (AutomotiveUI '18). Association for Computing Machinery, New York, NY, USA, 85-93. https://doi. org $/ 10.1145 / 3239060.3239071$

[26] Johan Merbah, Julien Jacquier-Bret, and Philippe Gorce. 2020. Interaction with a smartphone under different task and environmental conditions: Emergence of users' postural strategies. International fournal of Industrial Ergonomics 77 (2020).

[27] Kyle Montague, Hugo Nicolau, and Vicki L. Hanson. 2014. Motor-Impaired Touchscreen Interactions in the Wild. In Proceedings of the 16th International ACM SIGACCESS Conference on Computers \& Accessibility (Rochester, New York, USA) (ASSETS '14). Association for Computing Machinery, New York, NY, USA, 123-130. https://doi.org/10.1145/2661334.2661362

[28] Martez E. Mott, Radu-Daniel Vatavu, Shaun K. Kane, and Jacob O. Wobbrock. 2016. Smart Touch: Improving Touch Accuracy for People with Motor Impairments with Template Matching. In Proceedings of the 2016 CHI Conference on Human Factors in Computing Systems (San Jose, California, USA) (CHI '16). Association for Computing Machinery, New York, NY, USA, 1934-1946. https://doi.org/10. $1145 / 2858036.2858390$

[29] Martez E Mott and Jacob O Wobbrock. 2019. Cluster Touch: Improving Touch Accuracy on Smartphones for People with Motor and Situational Impairments. 
(2019), 27.

[30] Josip Musić, Daryl Weir, Roderick Murray-Smith, and Simon Rogers. 2016. Modelling and correcting for the impact of the gait cycle on touch screen typing accuracy. Mux the Fournal of Mobile User Experience 5, 1 (2016), 1.

[31] Alexander Ng, John Williamson, and Stephen Brewster. 2015. The Effects of Encumbrance and Mobility on Touch-Based Gesture Interactions for Mobile Phones. In Proceedings of the 17th International Conference on Human-Computer Interaction with Mobile Devices and Services (Copenhagen, Denmark) (MobileHCI '15). Association for Computing Machinery, New York, NY, USA, 536-546. https: //doi.org/10.1145/2785830.2785853

[32] Eunji Park and Byungjoo Lee. 2020. An Intermittent Click Planning Model. 1-13. https://doi.org/10.1145/3313831.3376725

[33] Bastian Schildbach and Enrico Rukzio. 2010. Investigating Selection and Reading Performance on a Mobile Phone While Walking. In Proceedings of the 12th International Conference on Human Computer Interaction with Mobile Devices and Services (Lisbon, Portugal) (MobileHCI '10). Association for Computing Machinery, New York, NY, USA, 93-102. https://doi.org/10.1145/1851600.1851619

[34] Afroza Sultana and Karyn Moffatt. 2019. Effects of Aging on Small Target Selection with Touch Input. ACM Trans. Access. Comput. 12, 1, Article 1 (Feb. 2019), 35 pages. https://doi.org/10.1145/3300178

[35] R. D. Vatavu, L. Anthony, and Q. Brown. 2015. Child or Adult? Inferring Smartphone Users' Age Group from Touch Measurements Alone. Springer International Publishing (2015).

[36] R. D. Vatavu, G. Cramariuc, and D. M. Schipor. 2015. Touch interaction for children aged 3 to 6 years: Experimental findings and relationship to motor skills. International fournal of Human - Computer Studies 74 (2015), 54-76.

[37] Chat Wacharamanotham, Jan Hurtmanns, Alexander Mertens, Martin Kronenbuerger, Christopher Schlick, and Jan Borchers. 2011. Evaluating Swabbing: A Touchscreen Input Method for Elderly Users with Tremor. In Proceedings of the SIGCHI Conference on Human Factors in Computing Systems (Vancouver, BC, Canada) (CHI '11). Association for Computing Machinery, New York, NY, USA, 623-626. https://doi.org/10.1145/1978942.1979031

[38] Daryl Weir, Simon Rogers, Roderick Murray-Smith, and Markus Löchtefeld. 2012 A User-Specific Machine Learning Approach for Improving Touch Accuracy on Mobile Devices. In Proceedings of the 25th Annual ACM Symposium on User Interface Software and Technology (Cambridge, Massachusetts, USA) (UIST '12). Association for Computing Machinery, New York, NY, USA, 465-476. https: //doi.org/10.1145/2380116.2380175

[39] Frank Wilcoxon. 1945. Individual Comparisons by Ranking Methods. Biometrics Bulletin 1, 6 (1945), 80-83.

[40] Jacob O. Wobbrock, Krzysztof Z. Gajos, Shaun K. Kane, and Gregg C. Vanderheiden. 2018. Ability-Based Design. Commun. ACM 61, 6 (May 2018), 62-71. https://doi.org/10.1145/3148051

[41] Jacob O. Wobbrock, Shaun K. Kane, Krzysztof Z. Gajos, Susumu Harada, and Jon Froehlich. 2011. Ability-Based Design: Concept, Principles and Examples. 3, 3, Article 9 (April 2011), 27 pages. https://doi.org/10.1145/1952383.1952384

[42] Julia Woodward, Jahelle Cato, Jesse Smith, Isaac Wang, Brett Benda, Lisa Anthony, and Jaime Ruiz. 2020. Examining Fitts' and FFitts' Law Models for Children's Pointing Tasks on Touchscreens. In Proceedings of the International Conference on Advanced Visual Interfaces (Salerno, Italy) ( $A V I$ '20). Association for Computing Machinery, New York, NY, USA, Article 56, 5 pages. https://doi.org/10.1145/ 3399715.3399844

\section{A TECHNICAL DETAILS OF THE ADAPTIVE TERNARY-GAUSSIAN MODEL}

\section{A.1 Similar Model}

The first step in constructing the DUDDS is to train the similar model. The similar model is a 2D Ternary-Gaussian model trained by a subset of the general dataset that has similar characteristics with the newly observed dataset.

In order to make the similar model has the characteristics from the specific circumstance while maintaining the general distribution attribute, we must find a subset in the general dataset that is most similar to the newly observed dataset. To do that, we use the model coefficients of the 2D Ternary-Gaussian model to represent features of each endpoint in the general dataset and select the subset based on these features.

As the model coefficients of the 2D Ternary-Gaussian model reflect the characteristics of a group of endpoints. In a special case that there is only one endpoint in the group, we can use the model coefficients to reflect the characteristics of that single endpoint. Therefore, we use the 2D Ternary-Gaussian model to reverse calculate model coefficients as the features of a single endpoint by substituting the endpoint into Equation (2) and (3). To substituting the endpoint $(x, y)$ into the Equation (2) and (3), we analogically replace $\mu_{x}$ and $\mu_{y}$ by $x$ and $y$, and replace $\sigma_{x}$ and $\sigma_{y}$ by 0 as follow:

$$
\begin{aligned}
& x=a_{x}+b_{x} V+c_{x} W \\
& y=0 \\
& 0=d_{x}+e_{x} V^{2}+f_{x} W^{2}+g_{x} \frac{V}{W} \\
& 0=d_{y}+e_{y} V^{2}+f_{y} W^{2}
\end{aligned}
$$

We cannot find the solution of Equation (6) because it has more unknowns than the equations. Therefore, when calculating one coefficient (e.g., $a_{x}$ ), we replace the other unknowns (i.e., $b_{x}, c_{x}$, $d_{x}, e_{x}, f_{x}, g_{x}, d_{y}, e_{y}$, and $f_{y}$ ) by model coefficients of the general model which was pre-fitted by general dataset:

$$
\begin{aligned}
a_{x}= & x-c_{x_{g e n l}} W-b_{x_{g e n l}} V \\
b_{x}= & \frac{x-c_{x_{g e n l}} W-a_{x_{g e n l}}}{V} \\
c_{x}= & \frac{x-b_{x_{g e n l}} V-a_{x_{g e n l}}}{W} \\
d_{x}= & -e_{x_{g e n l}} V^{2}-f_{x_{g e n l}} W^{2}-g_{x_{g e n l}} \frac{V}{W} \\
e_{x}= & \frac{-d_{x_{g e n l}}-f_{x_{g e n l}} W^{2}-g_{x_{g e n l}} \frac{V}{W}}{V^{2}} \\
f_{x}= & \frac{-d_{x_{g e n l}}-e_{x_{g e n l}} V^{2}-g_{x_{g e n l}} \frac{V}{W}}{W^{2}} \\
g_{x}= & \frac{-d_{x_{g e n l}}-e_{x_{g e n l}} V^{2}-f_{x_{g e n l}} W^{2}}{\frac{V}{W}} \\
d_{y}= & -e_{y_{g e n l}} V^{2}-f_{y_{g e n l}} W^{2} \\
e_{y}= & \frac{-d_{y_{g e n l}}-f_{y_{g e n l}} W^{2}}{V^{2}} \\
f_{y}= & \frac{-d_{y_{g e n l}}-e_{y_{g e n l}} V^{2}}{W^{2}}
\end{aligned}
$$

Where, $x$ and $y$ are the $\mathrm{x}$ and $\mathrm{y}$ coordinates of a endpoint of the newly observed dataset. $a_{x_{g e n l}}, b_{x_{g e n l}}, c_{x_{g e n l}}, d_{x_{g e n l}}, e_{x_{g e n l}}, f_{x_{g e n l}}$, $g_{x_{\text {genl }}}, d_{y_{\text {genl }}}, e_{y_{\text {genl }}}, f_{y_{\text {genl }}}$ are the model coefficients pre-fitted by general dataset. By doing this, we obtain a feature vector reflect distribution characteristics of that endpoint.

Given the definition of the feature vector, we use a $k$-means clustering algorithm [12] to cluster the endpoints in the general dataset into five groups by comparing the feature vectors of the endpoints and, select two of the five groups with the closest distance to the newly observed dataset. The number of groups of $k$-means clustering is the inflection point of the change trend of the sum of squares of the total inter-cluster distance with the increase of $k$ value. Since the endpoints in one group is insufficient to support fitting the Ternary-Gaussian model, two groups are chosen. The distance between two datasets is defined as the Euclidean distance between the mean feature vectors of the two datasets. Finally, the 
selected two groups of endpoints were used to train the similar model as shown in Figure 1 (a).

Note that, when performing the clustering algorithm, we actually did it separately for different target conditions in the general dataset. Only in this way can we ensure that the final selected endpoint groups contain endpoints in different target conditions evenly, which facilitates the fitting of the similar model.

\section{A.2 Gaussian Parameters Update}

Although the similar model already contains some characteristics similar to the newly observed dataset, in some cases, it may not learn any information from the newly observed dataset. For example, all endpoint groups in the general dataset are not similar to the newly observed dataset. Therefore, we further use a linear combination method to update the outputs of the similar model.

As we mentioned earlier, the similar model is actually a TernaryGaussian model. Given a target condition (i.e., target size $W$ and target speed $V$ ), the similar model outputs Gaussian parameters (i.e., $\mu_{\text {sim }}$ and $\Sigma_{\text {sim }}$ ) in that target condition (Figure $1(\mathrm{~b})$ ). For endpoints in the newly observed dataset, they are $\mathrm{x}$ and $\mathrm{y}$ coordinates of specified targets.

For updating the outputted Gaussian parameters with the endpoints in the newly observed dataset, as shown in Figure 1 (c), we use a linear combination with weights $1-w$ and $w$ to combine the Gaussian parameters produced by the similar model $\mu_{\text {sim }}$ (i.e., $\mu_{\text {sim }}$ and $\mu_{\text {sim }}$ ) and endpoint coordinates (i.e., $\mathrm{x}$ and $\mathrm{y}$ ):

$$
\begin{aligned}
& \mu_{x}=(1-w) \mu_{\text {sim }_{x}}+w x \\
& \mu_{y}=(1-w) \mu_{\text {sim }_{y}}+w y
\end{aligned}
$$

and the Gaussian parameters $\Sigma_{\text {sim }}$ (i.e., $\sigma_{\text {sim }}$ and $\sigma_{\text {sim }}$ ) and the deviation of endpoint coordinates from $\mu_{\text {sim }}$ (i.e., $x-\mu_{\text {sim }}$ and $\left.y-\mu_{\operatorname{sim}_{y}}\right)$ :

$$
\begin{aligned}
\sigma_{x}^{2} & =(1-w) \sigma_{\operatorname{sim}_{x}}^{2}+w\left(x-\mu_{\operatorname{sim}_{x}}\right)^{2} \\
\sigma_{y}^{2} & =(1-w) \sigma_{\text {sim }_{y}}^{2}+w\left(y-\mu_{\text {sim }}\right)^{2}
\end{aligned}
$$

We use $x-\mu_{\operatorname{sim}_{x}}$ and $y-\mu_{\text {sim }}$ to update $\sigma_{\text {sim }}$ and $\sigma_{\text {sim }}$ because they represent the offset of a endpoint from a distribution center, which corresponds to the standard deviation of the distribution. The weight of the linear combination $w$ is given by an SVM regression model. The aim of the SVM model is to find the most appropriate update weight in the linear combination, which will be elaborated later.

The above update happens each time a new endpoint from the specific user circumstance is observed and yields a new set of Gaussian parameters (i.e., $\mu$ and $\Sigma$ ) to the DUDDS. In cases that multiple endpoints in the same target condition are observed, the update generates multiple Gaussian parameters for one target condition. In this situation, we use the mean of all the generated Gaussian parameters as the final Gaussian parameters in this target condition. After repeatedly expanding the target condition and the corresponding Gaussian parameters in the DUDDS, the final Adaptive TernaryGaussian model which is trained by the DUDDS will approach the actual model of the specific user circumstance (Figure 1 (c)) Once the final Adaptive Ternary-Gaussian model is trained, it can predict the error rate of a specified target via multivariate normal cumulative distribution function (CDF).

\section{A.3 Determining the Weight of the Linear Combination}

Inputs of the SVM model to determine the weight of the linear combination are features listed in Table 5 . These features are constructed to reflect the difference of the endpoint distributions between the the general dataset and newly observed dataset.

To train the SVM model, we need to construct a dataset that contains ideal $w$ as labels and corresponding features listed in Table 5 as inputs. To get the ideal $w$, we randomly select a number of endpoints from a pre-collected dataset under a specific user circumstance and, run multiple times the process of building the Adaptive Ternary-Gaussian model when traversing the value of $w$ from 0 to 1 with an interval of 0.1 . Then, we select the $w$ that maximizes the prediction performance of the final Adaptive Ternary-Gaussian model as the ideal $w$. The corresponding features listed in Table 5 are determined once the endpoints in the pre-collected dataset are selected.

We can get a dataset that contains ideal $w$ as labels and corresponding features by repeating the above process. After there are enough samples in this dataset, we can use it to train the SVM regression model which outputs the most appropriated update weight $w$ by given the general dataset and the newly observed dataset. 
Table 5: The features as input data for the SVM regression model.

\begin{tabular}{|c|c|c|}
\hline Index & Category & Feature \\
\hline 1 & The Size of Dataset & the number of the endpoints in newly observed data \\
\hline 2 & & the number of target conditions in newly observed data \\
\hline 3 & Distance of Feature Vectors & $\begin{array}{l}\text { Euclidean distance of the feature vectors between the general model } \\
\text { and the similar model }\end{array}$ \\
\hline 4 & & $\begin{array}{l}\text { Euclidean distance of the feature vectors between the general model } \\
\text { and the model fitted by newly observed dataset }\end{array}$ \\
\hline 5 & & $\begin{array}{l}\text { Euclidean distance of the feature vectors between the similar model } \\
\text { and the model fitted by newly observed dataset }\end{array}$ \\
\hline 6 & Distance of Distribution & $x_{i}-\mu_{\operatorname{sim}_{x}}$ \\
\hline 7 & & $y_{i}-\mu_{s i m_{y}}$ \\
\hline 8 & & $\left(x_{i}-\mu_{\operatorname{sim}_{x}}\right)-\sigma_{\operatorname{sim}_{x}}$ \\
\hline 9 & & $\left(y_{i}-\mu_{s i m_{y}}\right)-\sigma_{s_{i m}}$ \\
\hline 10 & & probability density function $(P D F)$ of $x_{i}$ in $N\left(\mu_{s i m}, \sigma_{s i m}^{2}\right)$ \\
\hline
\end{tabular}

